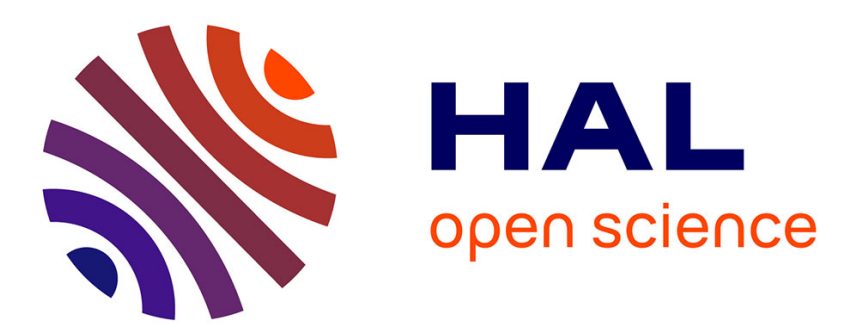

\title{
Les brûlages dirigés dans les Pyrénées-Orientales...de la régénération des pâturages d'altitude à la protection des forêts...
}

B. Lambert, V. Parmain

\section{- To cite this version:}

B. Lambert, V. Parmain. Les brûlages dirigés dans les Pyrénées-Orientales...de la régénération des pâturages d'altitude à la protection des forêts.... Revue forestière française, 1990, 42 (S), pp.140-155. $10.4267 / 2042 / 26128$. hal-03425354

\section{HAL Id: hal-03425354 \\ https://hal.science/hal-03425354}

Submitted on 10 Nov 2021

HAL is a multi-disciplinary open access archive for the deposit and dissemination of scientific research documents, whether they are published or not. The documents may come from teaching and research institutions in France or abroad, or from public or private research centers.
L'archive ouverte pluridisciplinaire HAL, est destinée au dépôt et à la diffusion de documents scientifiques de niveau recherche, publiés ou non, émanant des établissements d'enseignement et de recherche français ou étrangers, des laboratoires publics ou privés. 


\section{LES BRÛLAGES DIRIGÉS DANS LES PYRÉNÉES-ORIENTALES

\author{
... De la régénération des pâturages \\ d'altitude à la protection des forêts...
}

Dans les Pyrénées-Orientales, l'espace montagnard (300 à $2500 \mathrm{~m}$ d'altitude) représente un vaste espace d'environ 200000 ha sous influence de climats montagnards et méditerranéens. Sur cette terre, l'exode rural, qui a débuté dès la fin du XIX siècle, a eu comme conséquence une inadéquation des pratiques visant au renouvellement des ressources forestières et pastorales.

L'abandon de ces espaces a permis leur reconquête par des formations arborées pionnières: Pins à crochets en altitude, Chênes pubescents et Chênes verts plus bas. Dans les zones les plus dégradées, qui constituent généralement les parcours restants, la dynamique de la végétation est souvent bloquée au stade du Genêt purgatif en montagne ou du Ciste dans le piémont et constitue de ce fait une formidable accumulation de combustible tout en interdisant la mobilisation des ressources pastorales.

Aussi, les services publics gestionnaires de ces espaces (Office national des Forêts, Directions départementales de l'Agriculture et de la Forêt, etc...), ainsi que les élus sensibilisés par les incendies catastrophiques de 1976 et 1986, respectivement 17000 et 8000 ha, souhaitent protéger les massifs forestiers, et abaisser les dépenses liées à la lutte contre les incendies, en s'appuyant sur les acteurs locaux et surtout les éleveurs du département. Ces derniers, qui représentent plus de 500 familles avec 20000 unités de gros bétails, ont relancé cette activité en l'adaptant au nouveau contexte socio-économique local.

Pour cela, lors de rencontres de concertation entre les différents acteurs cités ci-dessus, auxquels il est bon d'associer les chasseurs et les services de secours et d'incendies, sont déterminés les choix d'aménagement du massif ou de la zone étudiée. C'est donc dans ce cadre qu'il peut être judicieux de proposer la création, au cœur ou à la périphérie des massifs forestiers, de coupures de combustibles exploitables par l'élevage. Mais face au fort embroussaillement, au fort pendage des parcelles, à la présence de rochers voire de murettes, qui interdisent généralement la mécanisation du débroussaillage, le brûlage dirigé selon des savoirfaire déjà éprouvés par ailleurs (USA, Portugal) apparaît souvent comme la dernière technique financièrement abordable.

Le brûlage dirigé s'insère alors, non seulement dans un système de gestion cohérent, mais surtout dans un plan d'aménagement où l'on fait un zonage des surfaces à débroussailler avant de décider du mode d'action à employer.

Nous verrons ainsi successivement:

- un exemple d'aménagement concerté où cette technique a pu s'inscrire : 
- la réalisation de deux chantiers d'altitudes:

- et enfin, au regard de notre petite expérience, nous proposons une espèce de règle de jeu ou de "check-list " pour bien appréhender les chantiers de brûlages dirigès.

\section{L'AMÉNAGEMENT DE LA FORÊT DOMANIALE DE NOHĖDES - URBANYA}

\section{Représentation}

\section{- Localisation}

Caractérisées par une pluviométrie faible (de l'ordre de $600 \mathrm{~mm} / \mathrm{an}$ ), les Pyrénées-Orientales se démarquent ainsi fortement du reste de la chaîne. Les altitudes bien que modestes ne permettent pas d'appréhender le caractère très accidenté du relief. Ainsi, la forêt domaniale d'Urbanya, d'une superficie de $535 \mathrm{ha}$, se développe de 800 à $1750 \mathrm{~m}$ d'altitude.

\section{- Historique}

L'exode rural et l'abandon des cultures constatés depuis le début du XXe siècle ont permis, ici, aux forestiers d'appliquer une politique d'acquisitions amiables sur les terrains "estimés être à vocation forestière ".

C'est ainsi que, de 1946 à 1989, furent acquis environ 500 ha (remembrement dès 1964).

Sur ces terrains exposés au Sud et à l'Est, l'incendie demeure depuis longtemps un problème. Dès 1950, les forestiers ont tenté de reconstituer les peuplements détruits par semis à la volèe sur les parcelles incendiées; mais en vain, la dégradation en landes à Cistes et à Genêts semble irréversible.

Pourtant, en 1974, le service RTM entreprend des reboisements intégraux visant à prévenir les risques d'érosion. De nombreuses essences furent utilisées (Pin noir, Cèdre, Douglas, Sapin de Nordmann, Épicéa, Pin sylvestre) à des densités de l'ordre de 10000 plants/ha.

Les mesures $\mathrm{DFCl}$ réalisées consistèrent en un réseau de pistes et quelques pare-feu (cf. cartes p. 143).

- État actuel

Faute de crédits, ni les plantations, ni l'infrastructure n'ont pu recevoir en temps voulu les entretiens indispensables:

- plantations non dépressées ;

- piste et pare-feu non entretenus.

\section{Problématiques}

- L'incendie de 1986: 1260 ha

Origine : criminelle dans la nuit du 19 au 20 juillet 1986.

Conditions météorologiques: longue période de sécheresse dans les jours précédant l'incendie. Forte tramontane.

Déroulement : parcours rapide sur Mosset durant la journée du 20 juillet ; boisements particuliers peu atteints, pistes et pare-feu + travaux préparatoires ayant fait coupe-feu.

Le 20 juillet: dans la nuit, le feu se développe sur Conat et prend deux directions: sud-est poussé par le vent vers Conat et Sainte-Marguerite et ouest vers la commune d'Urbanya.

Le 21 juillet au matin : le feu est arrêté sur les hauteurs de Conat mais progresse toujours en direction d'Urbanya dans une lande de Cistes à feuilles de laurier. Il atteint Coubere et La-Copie le soir. Durant la journée du 21 juillet, la piste pourtant embroussaillée en limite basse et le coupe-feu en limite Est ont servi à protéger les PC des terrains communaux. À noter le développement du front de feu selon une ligne de plus grande pente, ce qui facilitait la lutte le long de la piste.

Le 22 juillet: deux fronts; l'un sous le col de Vigues qui est contenu en partie haute (la partie basse est inaccessible et le feu descend sur Urbanya), l'autre à la rivière d'Urbanya, lui aussi inaccessible. 

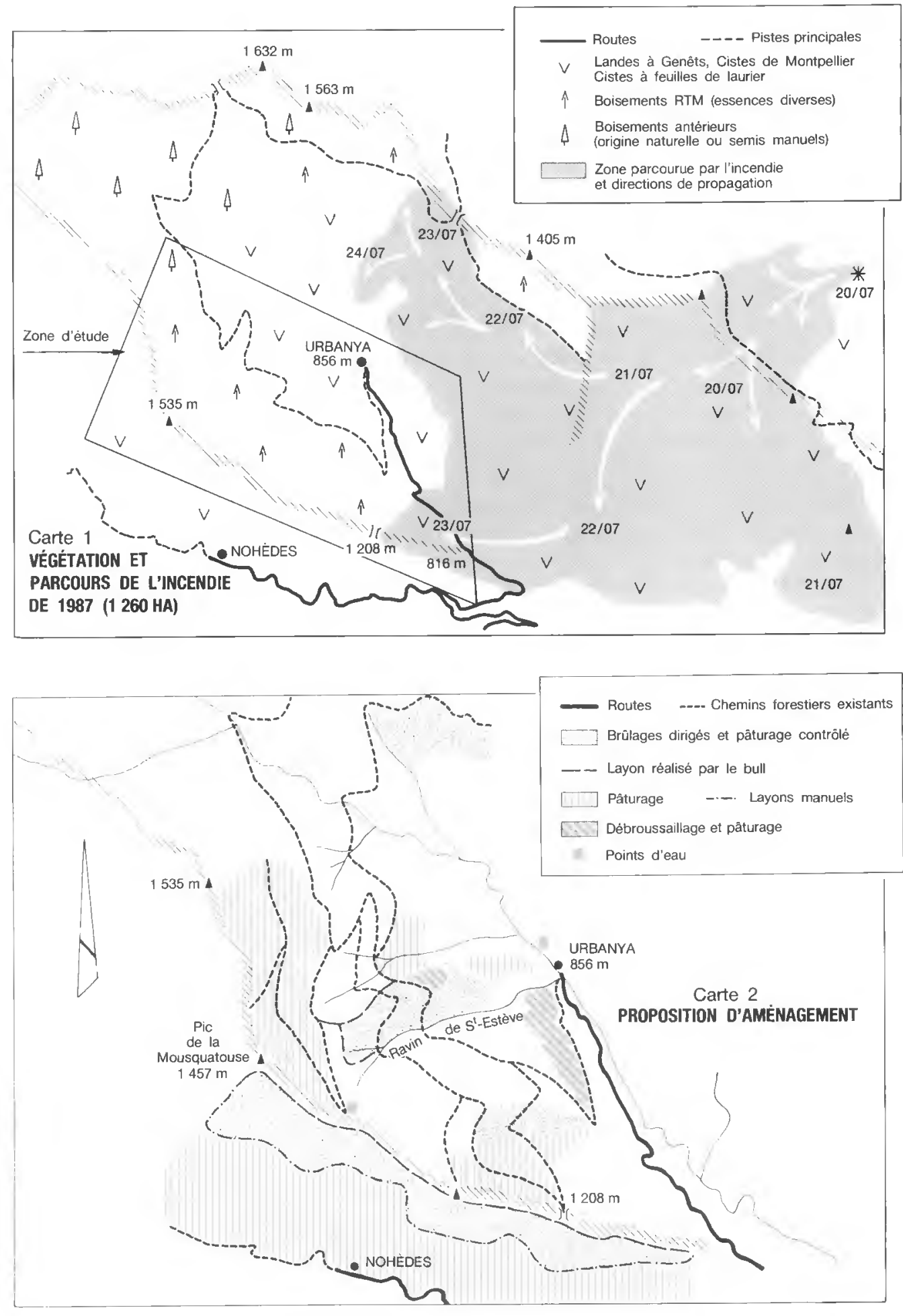
Dans la matinée, le feu saute la rivière et remonte en direction du col de Marsac dans la journée. Le soir à 20 heures : situation critique pour hommes et plantations - sans l'intervention des hommes de la Protection civile et des Canadair, dégâts importants aux plantations.

Nuit du 22 au 23 juillet: forts coups de vent qui réactive le front Nord, et depuis la cote 1150 environ, le feu remonte très rapidement vers le col des Vigues en brûlant les "Escausseils "; là aussi, catastrophe évitée de peu: le feu traverse les pistes et vient lécher plantations et forêts de Cobazet.

23 juillet: situation maîtrisée au Sud le matin; feu maîtrisé et éteint au-dessus du village vers 12 heures; front persistant sous les "Escausseils " dans une direction descendante. Durant la journée, l'ONF veille au col de Marsac pour éteindre des reprises effectives; sur le reste de ce front, situation claire.

Le 24 juillet: gros efforts dès le matin pour régler le plus grand des derniers fronts en rive gauche du ravin du Mantet. Feu éteint à 12 heures. L'ONF veille en partie haute (Secteur) et basse (Fsiran) ; situation claire définitivement à 20 heures.

\section{- Les enseignements tirés de l'incendie}

Le déroulement de l'incendie et la connaissance du terrain et de l'état des équipements ont permis aux gestionnaires de comprendre que :

- le risque d'incendie est sous-estimé: si les feux d'hiver ont des conséquences limitées du fait de la mauvaise combustibilité du Ciste à feuilles de laurier, le faible nombre d'éclosion d'incendie d'été ne doit pas faire oublier leur caractère généralement catastrophique ;

- la confiance dans l'efficacité des moyens de lutte traditionnels est remise en cause: comment intervenir précocement sur un départ de feux lorsque les délais de route sont supérieurs à une heure;

- les pare-feu sans entretien et les pistes insuffisamment larges ne permettent pas ici la progression aisée des moyens de lutte ni de ralentir durablement la progression du feu.

Donc en résumé, l'extinction d'un tel incendie est surtout le fruit de l'action des éléments météorologiques sur des configurations de feux favorables.

- Comment minimiser les risques de propagation d'un incendie?

C'est à cette question, éludée jusqu'ici, que nous allons tenter de répondre.

Le parti-pris choisi est de chercher à créer des zones de moindre accumulation de combustible rendant ainsi plus aisee l'intervention des moyens de secours.

\section{Propositions d'aménagement du massif}

- L'analyse des parcours des incendies passés (archives)

Elle permet de dégager deux types de progression :

- par tramontane et vent "tournant " dans le cirque d'Urbanya ;

- par vent du sud et feux en provenance de Nohèdes.

C'est suivant ces deux hypothèses que l'on a déterminé l'emplacement du dispositif. Le choix des techniques s'est effectué en fonction des conditions ponctuelles de chantiers. Nous avons ainsi retenu:

- débroussaillage mécanique sur 5 ha ( +15 ha hors domanial);

- décapage au bull et sursemis sur 4 ha;

- labour à la charrue à disque et remise en culture : 1 ha ;

- pacage des animaux : 50 ha ( +50 ha hors gomanial) ;

- brûlage dirigé plus pacage: 120 ha environ ( +100 ha hors domanial).

(Avantages et inconvénients de chaque technique ont déjà fait l'objet de développements dans la littérature forestière; seul le brûlage dirigé sera envisagé plus loin).

- La confrontation à la réalité

Ce projet, tel qu'il est conçu, ne prend en compte que la protection de la forêt domaniale contre l'incendie. Néanmoins, pour être applicable, il doit être soumis à l'approbation des différents 
utilisateurs reconnus. C'est ainsi qu'un consensus a pu s'établir entre forestiers, chasseurs, éleveurs, et habitants de la commune.

II a donc été choisi de ne réaliser qu'une version simplifiée du projet, centrée sur les terrains domaniaux et servant de tests et de démonstration en vue d'une extension ultérieure.

\section{QUELQUES EXEMPLES DE CHANTIERS}

\section{Les chantiers de brûlages dirigés du ravin de Saint-Estève}

\section{- Présentation}

II s'agit d'un milieu de versant plein Sud, de 40 ha environ, qui s'étage de 950 à $1300 \mathrm{~m}$ d'altitude. II est occupé par des communautés de Genêts purgatifs de plus de dix ans dont le recouvrement reste supérieur à $75 \%$. Dans les plages restantes subsiste une pelouse mésophile sur sol affleurant de schistes (Festuca ovina, Koeleria cristata, Agrostis alba).

La pierrosité de la pente supérieure à $60 \%$ interdit toute mécanisation ou débroussaillage. Par contre :

- les accès rendus aisés en tout temps, grâce au réseau de pistes forestières ;

- le cloisonnement du chantier en trois parcelles, réalisé en majeure partie par les pistes existantes, le ravin de Saint-Estève et les surfaces remises en cultures après débroussaillage;

- la proximité d'un point d'eau à moins de $2 \mathrm{~km}$;

tout cela rendait envisageable l'ouverture du milieu par les brûlages dirigés et ce, malgré l'importance du combustible (Genêt de 40 à $80 \mathrm{~cm}$ de haut, soit plus de $20 \mathrm{t} \mathrm{MS/ha).}$

- Objectif du traitement et type de brûlage à préconiser (figure 1 p. 146)

II s'agissait d'assurer la création d'une coupure de combustible qui puisse être entretenue par le pâturage automnal et printanier des bovins allaitants de l'exploitation limitrophe.

Le brûlage devait donc détruire la partie aérienne du Genêt purgatif, voire en brûler les éléments les plus fins, afin d'assurer un éclairement au sol susceptible d'entraîner la recolonisation du milieu, à partir des plages de graminées encore en place (voire par les sursemis).

Compte tenu de la nécessité de sauvegarder le tapis herbacé et par conséquence de limiter le choc thermique sur le sol, nous aurions pu retenir l'hypothèse d'un feu rapide et peu intense

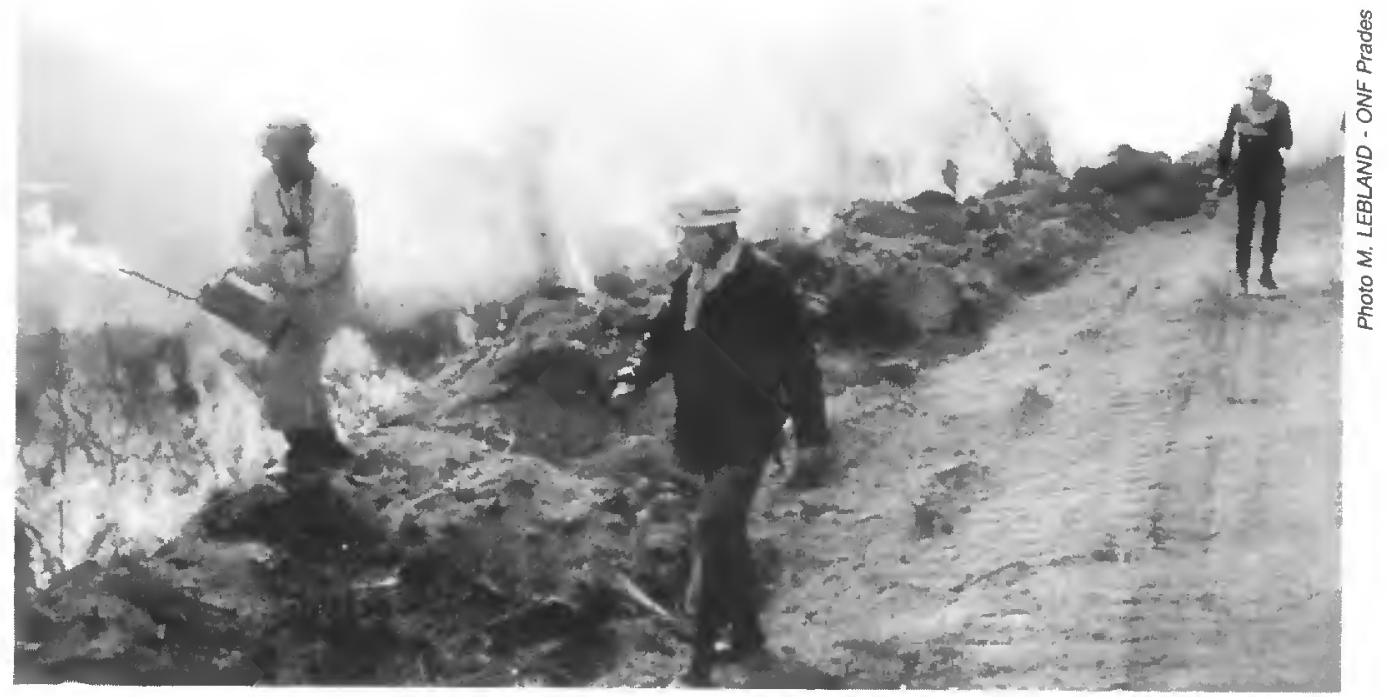

Réalisation d'une bande de sécurité en appui sur une bande terrassée au bulldozer (Urbanya 1990). 
(feu montant après une bonne pluie ou sur un manteau neigeux de quelques centimètres). Toutefois, l'importance de la pente, qui laissait augurer de la forte puissance du front de flamme et des sautes de feu possibles lièes à l'absence du contre-vent, et surtout la valeur des boisements limitrophes nous incitèrent à privilègier les aspects de sécurité sur les effets attendus du brûlage sur la pelouse. En conséquence, le dispositif visait à permettre la réalisation d'un feu périmétral, il nous a fallu pour cela:

- la confection de layon coupe-feu au bull sur certaines lisières ( $1200 \mathrm{~m}$ environ) pour finir de découper le versant en trois parcelles;

- réaliser le brûlage avec des conditions météorologiques (un temps froid, "air humide ", vent faible) susceptibles de freiner la montée en puissance du front de flamme;

- des établissements sur les bordures au vent;

- l'usage du feu à la recule ou à contre-vent pour ces mêmes bordures sur plus de $50 \mathrm{~m}$;

- et l'emploi du feu montant ou au vent une fois les sécuritès rèalisées.

- Réalisation (cf. figure 1)

Météo : vent nord-est 10 à $15 \mathrm{~km} / \mathrm{h} ; \mathrm{T}=10^{\circ} \mathrm{C}$; humiditè relative de l'air $<60 \%$.

État du milieu : sol $15 \%$ d'humidité ; litière $20 \%$; èléments fins des ligneux morts $15 \%$; Genêts verts $45 \%$.

Personnel: 2 cadres responsables de l'allumage ; 10 sapeurs pour la surveillance des bordures; $1500 \mathrm{~m}$ par parcelle.

Matériel: 2 CCFM ; un pour l'approvisionnement en eau (1) et l'autre pour l'établissement du dispositif préventif; deux fois $500 \mathrm{~m}$ de tuyau (22) pour l'établissement; 2 drip torch et 201 de mélange pour l'allumage ; 4 radios portatives.

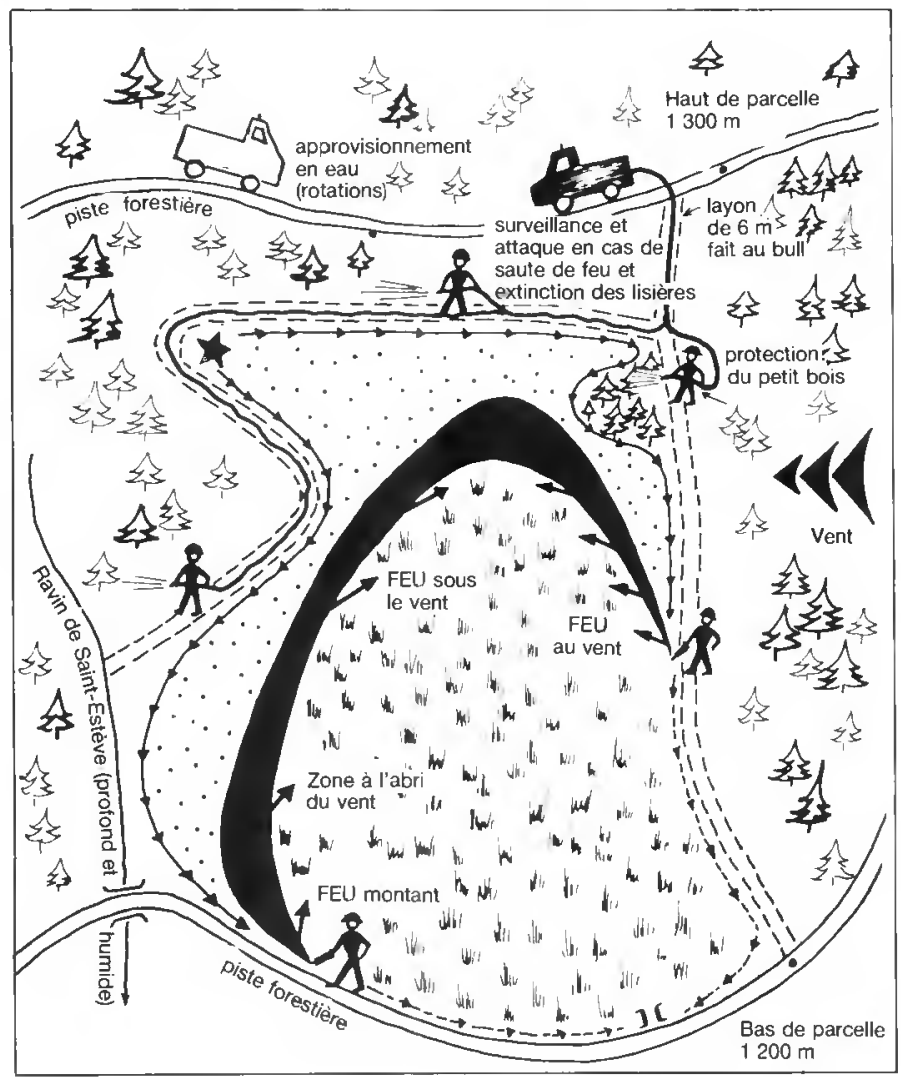

Figure 1 LE CHANTIER DE BRÛLAGE DIRIGÉ DU RAVIN DE SAINT-ESTĖVE (15 HA)

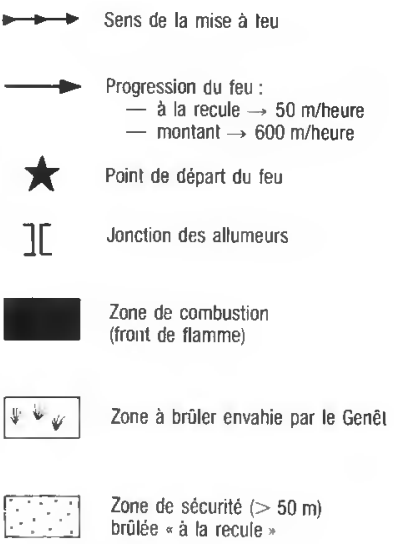

(1) Pour les services d'incendie, le choix de l'emplacement de la lance (ou des lances), le déroulement et le branchement de tuyaux, la mise en route du groupe moto-pompe constituent "l'ètablissement ". 
Durée des chantiers : 3 heures pour cette parcelle ( 30 ha dans la journée).

Caractéristiques du feu: $\mathrm{T}$ à $1 \mathrm{~m}>1000^{\circ} \mathrm{C}$, au sol $>600^{\circ} \mathrm{C}$; front descendant, $1 \mathrm{~m}$ de haut, continu $50 \mathrm{~m} /$ heure ; front montant 5 à $10 \mathrm{~m}$ de haut, par langues, $600 \mathrm{~m} /$ heure.

Effet du feu : voir chapitre suivant, p. 149.

Coût approximatif : préparation $12000 \mathrm{~F}$; encadrement $2 \times 1500 \mathrm{~F}$, moyens de sécurité $5000 \mathrm{~F}$; soit environ $20000 \mathrm{~F} \div 30 \mathrm{ha} \simeq 700 \mathrm{~F} / \mathrm{ha}$.

- Conséquences des techniques de conduite du feu sur le milieu

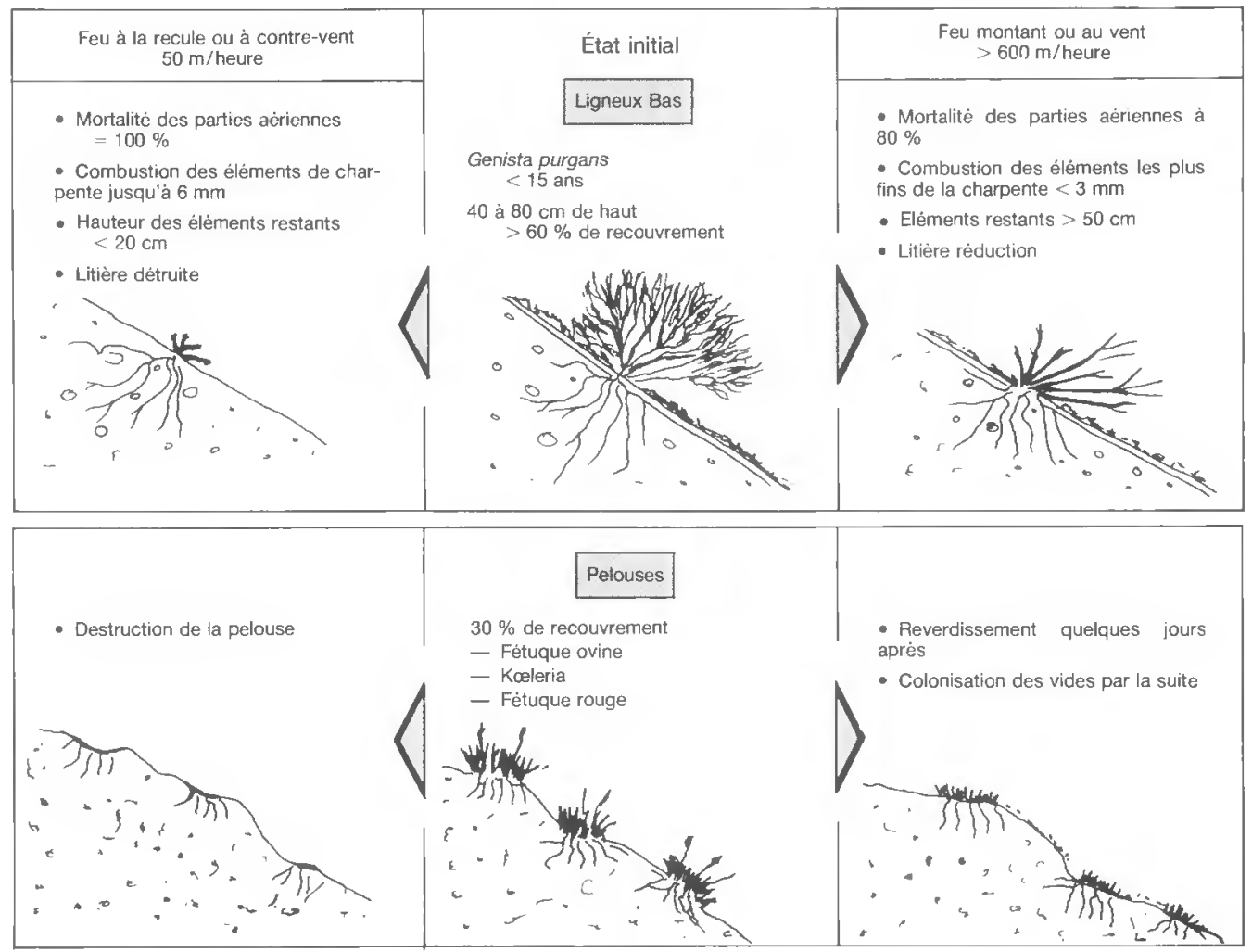

\section{Les chantiers de brûlages dirigés de la crête de la Mousquatouse}

\section{- Présentation et objectif de traitement}

Milieu et finalité semblable au ravin de Saint-Estève, toutefois le recouvrement de la pelouse est supérieur à $50 \%$. De plus, la topographie et la configuration des chantiers rendent peu probables des sautes de feu dans les boisements limitrophes: les aspects de sécurité ne sont plus prioritaires. Nous avons donc opté pour la recherche de conditions météorologiques et de milieu susceptibles d'autoriser un brûlage hivernal avec peu de moyens en hommes et matériels. Brûlage destiné à privilégier la régénération du tapis herbacé.

\section{- Préparation}

La préparation a impliqué la confection d'un layon. Confection de coupe-feu au bull sur la crête (sur $2 \mathrm{~km}$ environ), à la lisière des boisements. Ensemencement immédiat par les éleveurs de la bande travaillée.

Découpage des chantiers en 4 morceaux. Chaque parcelle représentant les possibilités de brûlage d'une petite équipe de 4 personnes durant une journée d'hiver. 


\section{- La réalisation}

Elle suppose de réunir les conditions et les moyens suivants (cf. figure 2):

Météo et condition locale: $T<5^{\circ} \mathrm{C}$, humidité relative $>50 \%$; vent nul ou faible de secteur nord-est à est... Une pluie quelques jours avant le brûlage, voire un sol gelé ou couvert de neige est souhaitable afin d'obtenir une humidité relative des éléments fins qui propageront le feu $<30 \%$ alors que les couches inférieures de la litière auront plus de $30 \%$.

Personnel: 2 cadres pour l'allumage +3 personnes pour la surveillance des lisières; seaux; pompes; pelles.

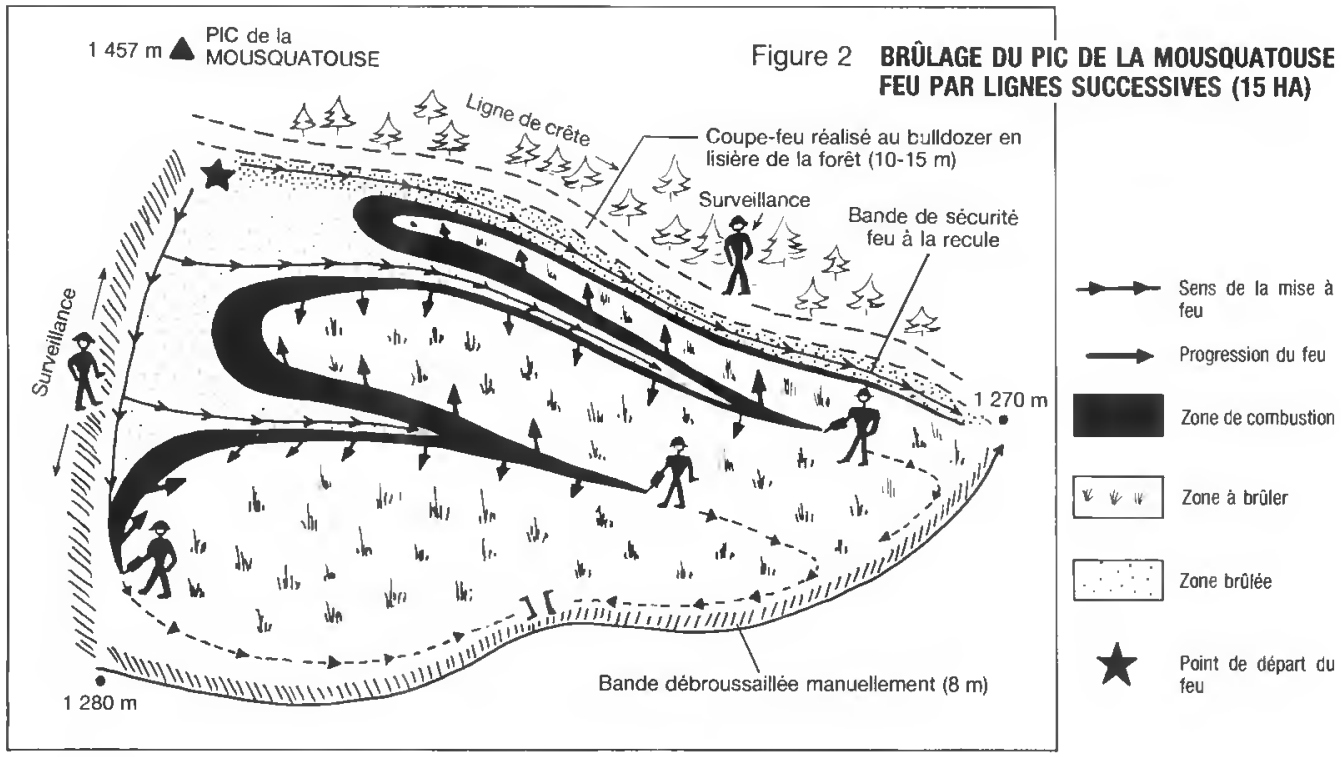

\section{- Coût approximatif pour 100 ha}

Layons coupe-feu et subdivision : $30000 \mathrm{~F}$; 5 journées avec 5 hommes : $30000 \mathrm{~F}$; soit $60000 \mathrm{~F}$ ou $600 \mathrm{~F} / \mathrm{ha}$.

\section{- Effet du brûlage}

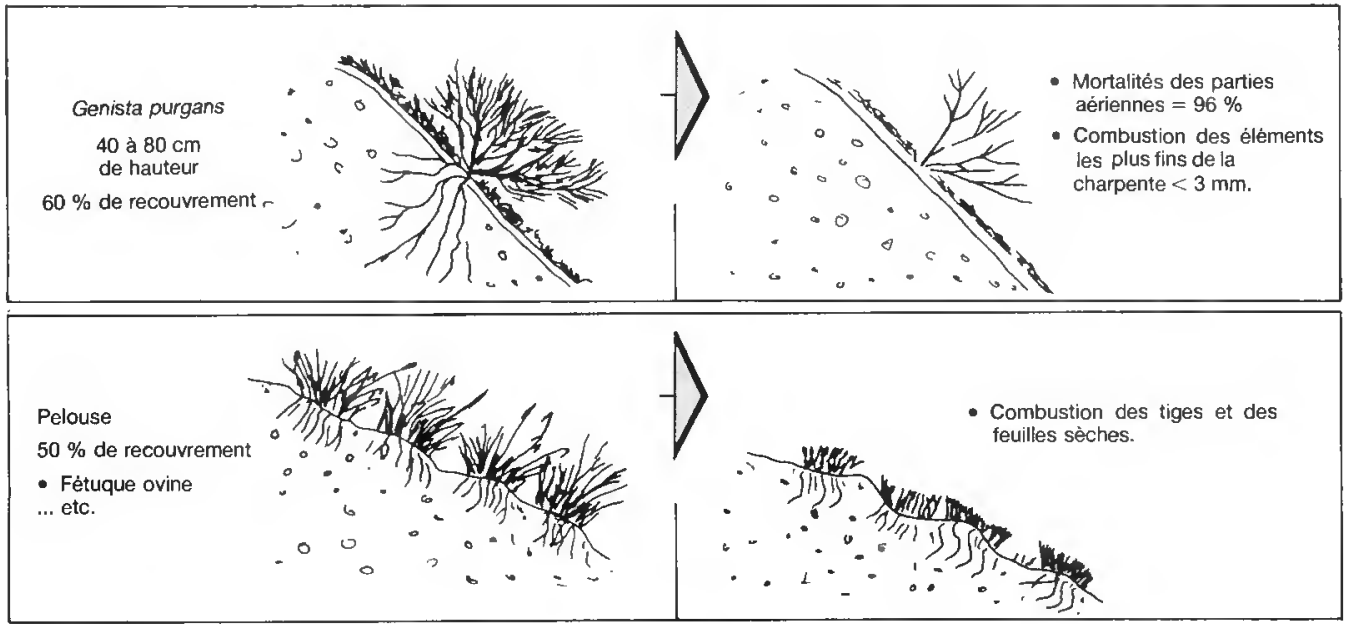




\section{PROCÉdURE DE RÉALISATION DES CHANTIERS}

\section{La check-list de la visite de terrain}

Au cours de ces visites, nous suivons le processus d'analyse logique suivant:

\section{1- Quelle est la cohérence de la demande?}

Objectifs de traitements, place de la parcelle dans le système d'exploitation, l'aménagement forestier ou le plan de lutte contre l'incendie. Quelle sera la gestion post-brûlage à court et à moyen terme? Comment prendre en compte les aspects cynégétiques et paysagers ? ... Cette étape est indispensable pour obtenir un consensus social local vis-à-vis des brûlages (à cette occasion peut émerger le problème de la maîtrise foncière).

\section{2- La végétation est-elle inflammable?}

L'inflammabilité qualifie la facilité avec laquelle les éléments fins de la litière et des espèces végétales en place vont prendre feu et assurer la propagation du front de flamme. Cette notion est capitale pour apprécier la facilité avec laquelle le brûlage pourra être conduit. Elle s'articule autour de trois critères:

- La nature des associations végétales et de la litière: en effet, plus un combustible est finement divisé et plus il sera inflammable (facilité d'échange de l'eau avec l'atmosphère, de dessiccation en avant du front de flamme et intensité du mélange combustible-comburant). Voici, à titre d'exemple, une classification d'inflammabilité de quelques associations végétales pour une teneur en eau de la litière et des éléments fins morts sur pied inférieure à $25 \%$ :

- Inflammabilité forte: pelouse de Molinie, de Fétuque spadicée, Fougères séchées sur pied.

- Inflammabilité assez forte : association où dominent (plus des deux tiers du recouvrement) soit l'Ajonc épineux, le Genêt purgatif, la Callune; les pelouses de graminées séchées sur pied de Fétuque scoparia, d'Agrostis, de Brachypodes.

- Inflammabilité modérée: pelouse rase de graminées, litière dense d'aiguilles de résineux (Pins, Genévriers), litières à grosses feuilles (Chênes blancs, Châtaigniers) ou à feuilles coriaces (Chênes verts, Chênes-liège, Lierre) ou à éléments de charpente de 3 à $6 \mathrm{~mm}$ de diamètre (Ronces, Églantiers), Chênes kermès, Genévriers.

- Inflammabilité faible: Cistaies pures ou dominantes, Genêt à Balai.

- Inflammabilité nulle: Pruneliers, Aubépines.

- Les conséquences de la pente et des vents dominants: dans la pratique, il arrive fréquemment de rencontrer à l'échelle de l'are, une juxtaposition d'associations peu et très inflammables. Dans ce cas, il faut se souvenir que la pente et le vent permettent de contrebalancer les conséquences du recouvrement insuffisant de l'association la plus inflammable (sous réserve d'une bonne dispersion et d'un recouvrement supérieur à $30 \%$ ). En effet, au-dessus du front, le courant de convection évacue plus de $80 \%$ des calories. Lorsque le feu monte une pente ou lorsqu'un vent le pousse, ce courant s'oriente au ras du sol. Ceci entraîne alors simultanément un dessèchement de la litière et des éléments les plus fins en avant du front de flamme, donc un accroissement de sa vitesse de propagation et surtout des transports de flammèches, voire de brandons, à plusieurs dizaines de mètres au-delà (sautes de feu). Ces effets, utilisés pour permettre de brûler des formations discontinues ou hétérogènes (cf. le cas précédent) posent par contre d'énormes problèmes de sécurité pour les brûlages de parcelles en milieu de versant.

- La période la plus favorable à préconiser pour avoir une teneur en eau minimale des éléments fins qui vont conduire le feu: bien que ce soit en période de repos de la végétation que les végétaux et à plus forte raison leurs éléments fins sur pied sont les plus inflammables (éléments vivants: moins de $50 \%$ de teneur en eau relative; éléments morts et litière : moins de $20 \%$, il est illusoire de croire que les brûlages dirigés peuvent être réalisés systématiquement de novembre à mars (la période estivale étant proscrite pour des raisons évidentes de sécurité ainsi que pour les effets destructeurs beaucoup plus importants sur le milieu). Le climat méditerranèen offre fréquemment des arrière-saisons douces et humides, favorables à une remontée de l'humidité par capillarité et au redémarrage de la végétation après le long arrêt lié à la sécheresse d'été. Aussi, pour entraîner une dessiccation suffisante des premiers horizons de la litière et des éléments fins morts ou vivants sur pied, une période de vent voire de gel est nécessaire après les précipitations (ceci est impératif pour la callunaie et les cistaies). 


\section{3- Cette formation est-elle combustible?}

La combustibilité caractérise la puissance du front de flamme issu d'une formation végétale donnée. En conséquence, elle détermine la nature et l'importance des moyens de sécurité à mettre en œuvre pour maintenir le front de flamme dans la parcelle (largeur du coupe-feu, surveillance des sautes de feu, etc...), et surtout le choc thermique potentiel sur les premiers horizons du sol (pour pondérer ce dernier, on pourra souhaiter une couverture neigeuse ou un sol gelé). La combustibilité dépend des espèces végétales en présence et de la structure de la végétation :

- La nature des espèces végétales: lors de la combustion, il est loisible de définir trois temps : l'évaporation (endothermique), la pyrolyse (endothermique) qui entraîne la combustion de gaz inflammables et la combustion de ces gaz (exothermique). Comme la quantité de chaleur consommée par l'évaporation de l'eau reste très importante, le pouvoir combustible de la biomasse est bel et bien fonction de sa teneur en eau. De plus, lors du passage du front de flamme, seule brûle la partie des appareils végétatifs où le mélange combustible-comburant est le plus intense et où, au demeurant, la teneur en eau relative est la plus faible car le plus sujet à la dessiccation. Ainsi, plus les feuilles et rameaux représenteront une masse combustible importante et plus le front du feu montera en puissance. À l'opposé, les végétaux faiblement divisés, à feuilles coriaces et/ou riches en eau seront peu combustibes tout en offrant une litière peu inflammable.

Le Guide technique du forestier méditerranéen (CEMAGREF, 1989) et notre petite expérience nous permettent de proposer la classification suivante en période hivernale de la combustibilité de quelques espèces (notée de 1 à 8 ) :

Ligneux hauts: 7- Chênes verts, Chênes blancs; 6- Oliviers, Pins.

Ligneux bas : 8- Ajoncs épineux et Genêts purgatifs ; 7-Genévriers, Callune, Bruyères, Calycotome, Genêt scorpion ; 6 - Ronces, Genêts à Balai, Romarin, Thym, Lavande ; 5 - Cistes à feuilles de laurier ; 3- Lierre, Salsepareille, Cistes de Montpellier ; 1 - Pruneliers, Aubépines, Églantiers. Herbacées séchées sur pied: 6- Fougères : 5- Grande Fétuque (spadicée, scoparia) et Molinie ; 4- Brachypode, Agrostis; 2- Fétuque ovine.

- Plus la structure de la végétation sera homogène et plus le front de flamme aura tendance à atteindre la puissance permise par les conditions météorologiques et l'état de sécheresse du milieu. En effet, le tlux de rayonnement émis par le "panneau radiant " du tront de feu est extrêmement intense à proximité immédiate. C'est donc ce rayonnement à courte distance (quelques mètres) qui cause le dessèchement et la pyrolyse du combustible en avant du front et assure la progression et la montée en puissance de ce dernier. Ainsi, une structure discontinue "cassera" la vitesse et la hauteur du front de flamme: par exemple, un front puissant ( 3 à $5 \mathrm{~m}$ de haut, $600 \mathrm{~m} /$ heure) issu d'une communauté dense de Genêts purgatifs se changera en petit feu extrêmement rapide dans une zone à graminées (pouvant atteindre 1,5 km/ heure). À l'opposé, nous avons observé régulièrement des phénomènes de mini-tornades de flammes dans les formations denses et régulières de Genêts avec entraînements de brandons à plusieurs dizaines de mètres de hauteur.

\section{4- Comment fractionner les chantiers et définir des parcelles?}

Comme nous venons de le voir, les associations végétales, leur état physiologique, la teneur en eau relative de leurs éléments fins et les conditions méléorologiques du jour (vent, température, etc...) déterminent l'inflammabilité et la combustibilité de la végétation á un instant donné. De ce fait, les conditions réclamées pour réaliser un brûlage dans la cistaie pourront fort bien être défavorables dans la callunaie limitrophe, etc...

De plus, sur des milieux où la végétation peut être homogène, il est fréquent de rencontrer des situations topographiques fort différentes. Ainsi, les contrastes d'ensoleillement et de flux des vents qui en découlent sur la même parcelle vont créer des différences notoires d'humidité de la litière et des éléments fins. II en sera de même pour l'orientation et la force du vent. Donc, multiplication des difficultés pour conduire et maîtriser le front de feu. Tout incite à fractionner les chantiers en autant de parcelles homogènes. Cette homogénéité est définie par:

- l'unité topographique: milieu, bas, haut de versant, exposition...

- l'unité écologique: le faciès de végétation (espèce dominante par strate, ligneux hauts, bas et herbacées). 
Pour chaque parcelle sera reprécisé alors l'effet attendu du feu sur les différentes strates et en conséquence les types de brûlage possibles.

C'est ici que le feu reprend sa place dans la gestion du territoire. II redevient avec les autres techniques parcellaires (phytocides, travaux du sol) un moyen de restructurer l'espace en autant de terroirs.

\section{5- Les types de brûlage à préconiser pour chacune des parcelles}

Selon la finalité du brûlage, il s'agira, soit de minimiser les effets du front de flamme sur les appareils aériens de la strate arborée, soit d'atténuer seulement le choc thermique sur le tapis herbacé et les premiers horizons organiques du sol, soit tout simplement de limiter avant tout les risques de voir le feu échapper à tout contrôle.

Pour maîtriser ainsi les brûlages, les opérateurs bénéficient de 4 leviers:

- Le dispositif de sécurité : ce volet important découlera du type de brûlage à préconiser et des conditions météorologiques et d'état du milieu prévisibles ou souhaitables pour le jour du chantier. Souple et modulable, il est l'aboutissement de la démarche, nous le traiterons dans le paragraphe suivant.

- Les données météorologiques avant et pendant le brûlage : les données recueillies avant le brûlage sont celles qui influent directement sur la teneur en eau de la litière, des éléments fins et du sol, à savoir: les précipitations (nature, hauteur), températures (gel...) et vent (évaporation, accumulation de la neige, etc...). Pour les données du jour, il s'agit essentiellement des données qui contribuent à la maitrise du front de flamme: vent (orientation, puissance $\leqslant 20 \mathrm{~km} /$ heure), température maximale $\left(\leqslant 15^{\circ}\right)$, humidité relative de l'air (30 à $100 \%$ selon les chantiers).

- La conduite du feu: elle permet de corriger ou d'accentuer les conséquences de l'inflammabilité et de la combustibilité. Elle est donc liée aux deux leviers précédents. Elle s'appuie sur trois composantes:

- La méthode d'allumage : linéaire ou par points, ou lignes successives ou simultanées, voire en anneaux concentriques, etc... En l'absence de vent, chaque méthode crée une dynamique du courant de convection et du contre-vent spécifique (cf. Guide technique du forestier méditerranéen).

- La direction de propagation du front de feu par rapport au vent ou à la pente : sens du vent, à contre-vent, perpendiculaire.

- Le pourcentage de surface brûlée: brûlage homogène en une seule fois, découpage de la parcelle en plusieurs chantiers espacés de plus d'un an, brûlage par taches selon l'inflammabilité des associations végétales (on revient au cas présent), brûlage par langues, en utilisant les rafales de vent, etc...

Ces trois composantes entrent en jeu pour définir la configuration du front de flamme. On pourra ainsi rencontrer des fronts présentant les caractéristiques suivantes:

1 - Propagation dans le sens du vent ou de la pente: feu puissant, peu intense, mais très rapide ( $>200 \mathrm{~m} /$ heure) à très large, et très haut front de flamme.

2 - Propagation à contre-vent (ou à contre-pente) : feu peu puissant, mais intense et très lent $(<50 \mathrm{~m} /$ heure) à étroit et bas front de flamme.

3 - Propagation dans une direction perpendiculaire à celle du vent ou de la pente : feu à caractéristiques intermédiaires: le choix de la technique dépend de l'objectif de brûlage. Pour l'entretien de sous-bois, on optera pour le type 2 ; par contre, pour favoriser les herbacées, on préfèrera le type 1 ou 3 (dans la mesure où la teneur en eau des premiers horizons du sol est suffisante).

- La teneur en eau des couches inférieures de la litière et des premiers horizons du sol : il s'agit de limiter les effets du choc thermique sur les appareils souterrains des végétaux, voire le plateau de tallage des graminées. Pour cela, un manteau neigeux, le gel ou l'évaporation de l'eau des premiers horizons constituent le meilleur effet tampon. Une visite la veille ou l'avantveille est donc indispensable. II va de soi que données météorologiques, conduite du feu, état du milieu ont des effets synergiques sur le milieu. Aussi, afin de donner un aperçu de la synergie existante, nous proposons quelques données recueillies sur une formation méditerranéenne. II s'agit d'une cistaie (Ciste de Montpellier, Ajonc épineux et Brachypode rameux). Cet exemple est d'autant plus intéressant que le Ciste s'avère être un bon indicateur du niveau de 
Figure 3

UN EXEMPLE DES EFFETS DIVERGENTS DES TYPES DE BRÛLAGE SUR L'ÉVOLUTION D'UN MILIEU (CISTAIES)

choc thermique qu'a pu recevoir le sol. En effet, la levée de dormance de ces graines est proportionnelle au flux calorifique reçu: $100 \%$ de germination sont observés après un passage au four à $130^{\circ} \mathrm{C}$ durant quelques minutes (au-delà, la température létale est atteinte, en deçà le pourcentage chute) (Oustric, 1985). Sur la figure 3 (ci-contre), nous avons mis en parallèle, selon les types de brûlage, les courbes pluriannuelles de germination du Ciste : elles indiquent le niveau du choc thermique et in fine le salissement du milieu et l'évolution du tapis herbacé. Évolution suivie d'après la méthode CEPE-INRA des bandes de lecture. Le nombre de contacts des graminées peut être assimilé à un volume sur pied. La lecture du schèma fait apparaître nettement que :

- Si les différentes techniques de conduite du brûlage semblent avoir la même efficacité pour ouvrir le milieu en détruisant la partie aẻrienne des ligneux bas, jugés indésirables, leurs effets secondaires, par l'importance du choc thermique induit sur les premiers horizons du sol sont fort différents. Ainsi, les feux descendants ou à la recule sur des sols secs favorisent la germination des Cistes et des Ajoncs épineux, les feux montants (ou en rateau en conditions plus humides) semblent à terme plus favorables aux graminées. Dans ce dernier cas, les Cistes sont trop peu nombreux pour entrer en concurrence avec la pelouse.

- Si le brûlage a pu mettre le sol à nu (absence de gelée, de neige ou de pluie récentes), dans la plupart des cas, le choc thermique est bien trop faible pour détruire le rhizome des graminées pérennes. Six mois après, le tapis herbacé a retrouvé son niveau initial (dans les cas les plus défavorables, le brûlage en taches ou par petites parcelles autorise une recolonisation par les franges).

\section{6- Le dispositif de sécurité à mettre en œuvre}

Ce dispositif est arrêté en deux étapes:

- Première étape : à la fin de la première visite de terrain, une fois les types de brûlage arrêtés, il est alors nécessaire d'intégrer les éléments qui vont contribuer au maintien du front de flamme dans la parcelle. À cet effet, on étudie quels sont:

- les accès : pour les équipes d'allumeurs, pour les moyens de sécurité ;

- la localisation des points d'eau... ou ceux qui peuvent être créés conjoncturellement ;

- les barrières existantes ou les points d'appui : routes, chemins, sentiers, ruisseaux, barre rocheuse, murettes, habitat, forêt ; 
- les alliés d'un jour: combe à neige, zone de congères ou de corniches, mouillères, lieu de changement du niveau d'inflammabilité et de combustibilité (talweg, crête, lisière de forêt), vent dominant ;

- les points faibles: zones à protéger (bois, maisons, vignes), surfaces où le feu montera en puissance ou s'accèlèrera, zone de vent.

L'aboutissement de la démarche conduira à préconiser la création d'éléments passifs, complémentaires : layon confectionné à la main ou au bull, amélioration des accès, brûlage de sécurité par temps pluvieux, etc...

Toutefois, comme le montre la figure 4, ci-dessous, les barrières ne sont jamais absolues. L'état de sécheresse du milieu, les conditions météorologiques locales vont décider de la dynamique du feu. C'est donc cette dynamique qu'il faut anticiper afin d'adapter le dispositif aux besoins.

Figure 4 LE DISPOSITIF DE SÉCURITÉ ET QUELQUES POINTS D'APPUI \& NATURELS *

\begin{tabular}{|c|c|c|}
\hline & $\begin{array}{l}\text { CONDITIONS FAVORABLES } \\
\text { état du milieu, type de feu, métèo } \\
\text { rendant la barriere suffisante }\end{array}$ & $\begin{array}{l}\text { CONDITIONS DÉFAVORABLES } \\
\text { les points d'appui doivent être complétés par un } \\
\text { dispositif adéquat }\end{array}$ \\
\hline $\begin{array}{l}\text { (1) BARRE } \\
\text { ROCHEUSE }\end{array}$ & $\begin{array}{c}\text { neige ou } \\
\text { zone humide }\end{array}$ & $\begin{array}{c}\text { FRONT MONTANT } \\
\text { courant de convection } \\
\text { (effets cheminees } \\
\text { chutes de pierre } \\
\text { sautes de feu) }\end{array}$ \\
\hline $\begin{array}{l}\text { (2) TALWEG- } \\
\text { RUISSEAU }\end{array}$ & 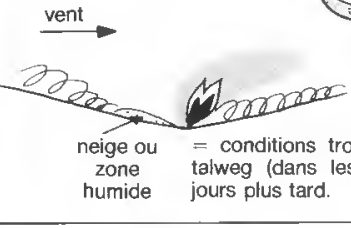 & sèches et reprise dans le \\
\hline $\begin{array}{l}\text { (3) COUPURE DE } \\
\text { COMBUSTIBLE: } \\
\text { GHEMINS, } \\
\text { LAYONS, } \\
\text { PLAOUES DE } \\
\text { NEIGE } \\
\text { a) en crête }\end{array}$ & Na & $\begin{array}{l}\text { FEU à la } \\
\text { RECULE }\end{array}$ \\
\hline $\begin{array}{l}\text { b) milieu de } \\
\text { versant }\end{array}$ & & \\
\hline c) ravins & humide & \\
\hline
\end{tabular}


En conséquence, les moyens de sécurité, tout en s'appuyant sur des chantiers préparés, doivent valoriser au mieux les "alliés" naturels. Le dispositif de sécurité prévu doit donc être modulable.

- Deuxième étape: la veille ou l'avant-veille, la dernière reconnaissance: outre l'état de sécheresse du sol et de la litière, il permet de faire le choix des moyens en hommes et matériels nécessaires (allumage, conduite, maîtrise, surveillance).

Bien que chacun des chantiers présente en matière de dispositif de sécurité sa propre spécificité, le nombre de personnes actives présentes ne doit jamais être inférieur à quatre et comporter au moins un ouvrier ou pompier supplementaire par tranche de 3 ha; au-delà de 10 ha, prévoir, dans tous les cas, selon la taille des chantiers: un ou plusieurs véhicules d'attaque (DANGEL ou CCFM). De plus, la mise à feu ne peut se faire que de jour, par vent régulier $(<20 \mathrm{~km} /$ heure) ou temps calme et le front de feu doit être surveillé en permanence jusqu'à l'extinction complète, même si cette extinction ne peut être obtenue que de nuit.

\section{La réalisation du brûlage}

La réalisation de chaque chantier s'appuie en fait sur deux tâches complémentaires : la conduite du feu et la surveillance des lisières pendant et surtout après le passage du front de flamme.

- La conduite : elle est l'aboutissement du raisonnement précédent auquel viennent s'ajouter les conditions météorologiques du jour. Elle définit donc le type d'allumage: localisation, distance entre les lignes, nombre d'allumeurs, vitesse de la mise à feu.

Moyens à prévoir par chantier

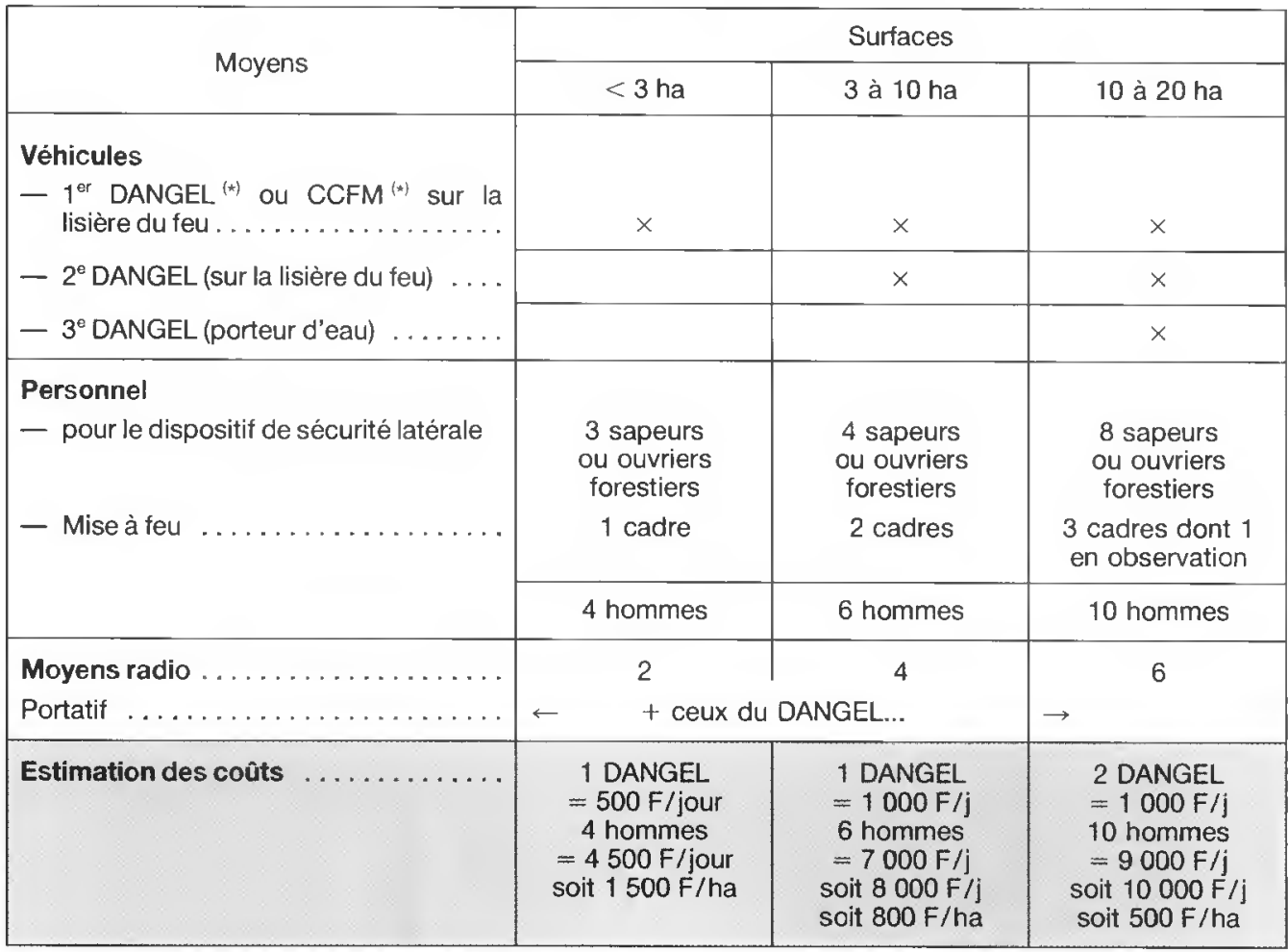

Au-delà de 20 ha, travailler soit avec la neige (zone d'altitude) ou avec les sections feux de forêt des UllSC.

() DANGEL = (équipage 2 hommes: cf. rubrique personnel) +500 I d'eau + moto-pompe +2 seaux pompes +2 battes a feu CCFM = (équipage 4 hommes : cf. rubrique personnel) +1200 I d'eau +500 m d'établissement possible. 
- La surveillance : elle est le fait de tous. Toutefois, dans le cas où la flamme se couche sur les coupures de combustible, il faut prévoir du personnel en plus sur les bordures dangereuses. L'importance de la surveillance est fonction des conditions locales, de la longueur et de la nature des coupe-feu.

Toutes ces tâches complémentaires doivent être réalisées par une équipe soudée et entraînée. À titre indicatif et en conditions optimales, le tableau ci-contre, p. 154 donne une idée des moyens à prévoir.

\title{
CONCLUSIONS
}

Les brûlages ne sont pas une fin en soi, et les demandes ponctuelles dites d'écobuages sont souvent les révélateurs d'une mauvaise gestion ou affectation du territoire.

Aussi, au regard du bilan des campagnes écoulées, 1500 ha depuis 1987, les brûlages dirigés apparaissent de plus en plus comme l'aboutissement d'un processus d'analyse logique. Processus qui prend en compte, la lutte contre l'incendie, la protection des boisements, les besoins des pasteurs comme des autres utilisateurs et gestionnaires de l'espace "montagnard".

C'est donc dans la définition d'un plan d'aménagement du massif, où l'élevage n'est plus oublié, que le feu peut retrouver sa place de simple élément technique de gestion parcellaire, au même titre que le labour, le débroussaillage, le défonçage, l'élagage, etc...

Parallèlement à cette volonté, cette procédure de concertation devient généralement un lieu où peuvent s'exprimer ouvertement les besoins des pasteurs en matière "d'écobuage ". De ce fait, la reconnaissance de cette technique, qui s'inscrit souvent dans un système de cultures cohérent et dont le maintien est lié à son efficacité et à sa "productivité ", permet de canaliser et, par conséquent, de limiter les "feux clandestins " qui sont à l'origine de nombreux incendies. D'une attitude répressive, nous sommes passés à une attitude d'accompagnement, et d'encadrement afin d'éviter toute banalisation de la technique.

Car, si le brûlage dirigé apparaît comme un moyen peu onéreux pour créer des coupures de combustible, ou pour participer au maintien des ressources pastorales, voire à l'entretien des forêts, il ne peut être mis en œuvre que par des équipes spécialisées. Équipes capables d'appréhender le chantier, d'être opérationnelles les jours où les conditions locales sont favorables, et surtout de conduire le feu pour en minimiser l'impact sur l'environnement, d'en interdire tout dérapage et surtout d'atteindre les objectifs agronomiques assignés.

\author{
B. LAMBERT \\ Ingénieur Recherche-Développement \\ SIME - SOCIĖTĖ O'ĖLEVAGE DES PYRÉNÉES-ORIENTALES \\ Avenue de la Gare \\ 66500 PRADES
}

V. PARMAIN

Chargé d'Études à la Division Prades-Capcir

OFFICE NATIONAL DES FORÉTS

32, allèe Arago

66500 PRADES 